\title{
Chromatography in Pharmaceutical Sciences in Brazil
}

Brazil is a country with continental dimensions. We have rain forest, deserts and mountains and a huge diversity of human and material resources. The wide mixture of cultures we have, has transformed Brazil into a peculiar country, with many different characteristics. The country, not only for its size, but also by its performance in various areas, represents important leadership in South America. In the academic area partnerships of cooperation with European and Latin American countries have increased the national scientific production. The post-graduate programs, with average annual growth estimated at $12 \%$, have a key role in the scientific literature of our country. Brazil currently contributes approximately $2 \%$ of the scientific world production, having produced over 19,000 scientific papers in 2007.

When I was invited as special editor of this issue of Chromatographia, I felt honored but I was also concerned to include manuscripts from all regions and as many areas as possible.

As a result of this invitation, I am pleased to see in this special issue papers from all over the country, including various areas of knowledge. The development of methods for the determination of drugs in pharmaceutical formulations or in biological fluids by means of modern techniques is included.
The application of these techniques in the field of pharmacognosy, including the synthesis of a stationary phase used in the separation of flavonoids was also covered as well as chromatographic applications in the field of toxicology.

We would like to thank all contributors hoping that this issue will serve as a stimulus for new submissions and wish everyone pleasant and interesting reading.

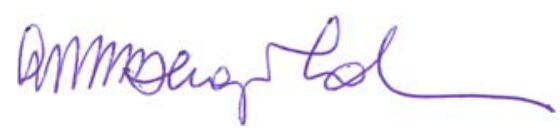

Ana M. Bergold 